\title{
Genesis of booster curves in Electric Power Assistance Steering Systems
}

\author{
V. Ciarla, V. Cahouet, C. Canudas de Wit and F. Quaine
}

\begin{abstract}
The typical architecture of an Electric Power Assistance Steering (EPAS) system includes a static map to provide the correct amplification to the driver's exerted torque. In literature, it is generally known as booster curve. This paper concerns the study of the amplification criteria, that are commonly used to these booster curves. The basic concepts of the Electric Power Steering (EPS) systems with a realistic model for the friction contact, that acts on the wheels are discussed. A relation between the assistance and the driver's torque is provided, under the hypothesis of a position-oriented control of the movement and the Stevens' power law. Finally, the simulation results proposed at the end of this paper validate the shape of the booster curves and are in accord with the initial hypothesis.

Index Terms-Electric Power Steering (EPS) system, Dahl friction model, static optimization.
\end{abstract}

\section{INTRODUCTION}

Nowadays, vehicles have been widely equipped with power steering systems and the way of providing assist torque is various. The EPAS systems have the tendency to replace the hydraulic power steering, due to better fuel consumption [1], [2]. They include the mechanical model of the steering column and the power steering unit. Moreover, this last element has the aim to reduce the steering efforts to a reasonable level, to ensure the most possible road feel feedback to the driver and to improve the return-to-center performance of the steering system.

The assistance provided by the electric motor of EPS systems reproduces the hysteresis cycle of the hydraulic valve, as shown in Fig. 1: the amplification torque $U$ grows, according to the value of the driver's torque $\tau_{v}$ and the vehicle speed $V$, then saturates. If the driver's torque changes in direction, we have the downhill phase of the amplification torque. During this phase, the amplification torque decreases, until reaching the lower saturation values. In literature, we can find many stationary models for the EPS system ([3], [4], [5], [6], [7],[8], [9], [10], [11]), where the hysteresis cycle is approximated by a single curve. Nevertheless, these investigations do not provide ground foundations (other than these curves reproduce the behaviour of the older power steering systems, based on hydraulic valves), neither evidence that they are optimal for the driver in any sense. This work aims

This work was partially funded by the project VolHand ANR-09-VTT-14. Valentina Ciarla, Violaine Cahouet, Carlos Canudas-de-Wit and Franck Quaine are with the Laboratoire Automatique de Grenoble, UMR CNRS, Grenoble 5528, France (e-mails: valentina.ciarla@gipsa-lab.grenoble-inp.fr, violaine.cahouet @gipsa-lab.grenoble-inp.fr, carlos.canudas-de-witegipsa-lab.grenoble-inp.fr, franck.quainedgipsa-lab.grenoble-inp.fr)

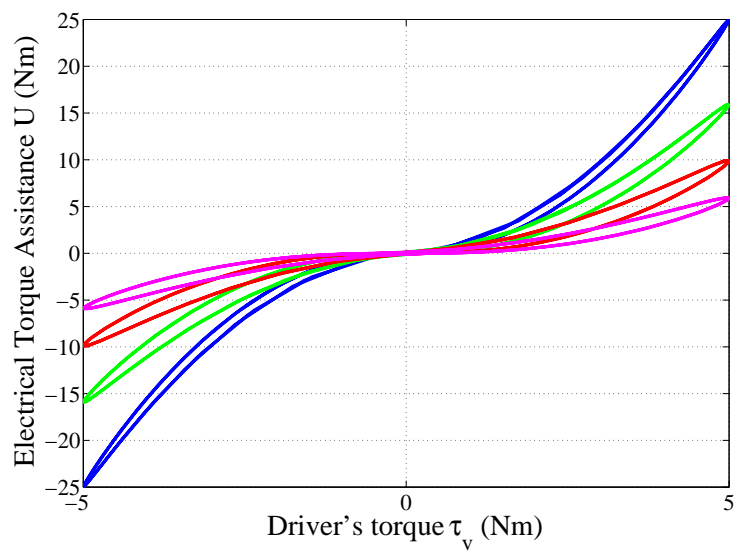

Fig. 1. Example of the booster curve, provided by the EPAS system, reported in [3].

for providing some rationality to these curves and validating their optimality for the drivers.

To this aim, it is interesting to refer to the literature concerning the criteria at the basis of the human coordination, that try to explain the human movement by minimizing a cost function. For example, to optimize the muscular effort during a one degree-of-freedom repetitive exercise motion, authors in ([12], [13]) choose to maximize the power spent by the user. To explain the trajectory formation in a constrained point-to-point motion, in [14] authors propose a combined criterion minimizing the variation of the hand contact force and the change of the actuating force over the course of the movement. In the case of a planar horizontal movements with one mechanical degree of freedom [15], the authors find an analytical expression for the predicted minimum torque change trajectories. In [16], the cost function is the square of the magnitude of jerk (rate of change of acceleration) of the hand integrated over the entire movement. The hypothesis at the basis of this paper is that driver's coordination provide a steering wheel movement that minimize the jerk criterion. Solving this optimization, the optimal steering wheel trajectories are derived. These trajectories allow to retrieve the driver's and the amplification torques, that model the shape of the booster curves.

The paper is organized as follows: in Section II, the main equations describing the EPAS system are introduced and the amplification model is defined; in Section III, the optimization problem, that will predict the optimal booster curves, is developed. Finally, in Section IV, significant simulation results and the conclusions are discussed. 
TABLE I

CONSTANT PARAMETERS OF THE EPSS

\begin{tabular}{|c|c|c|}
\hline Symbol & Description & Value \\
\hline \hline$J_{v}$ & Steering wheel inertia & $0.025 \mathrm{kgm}^{2}$ \\
$J_{m}$ & Motor inertia & $0.0004 \mathrm{kgm}^{2}$ \\
$J_{c}$ & Column inertia & $0.04 \mathrm{kgm}^{2}$ \\
$J_{w}$ & Rack inertia & $0.000784 \mathrm{kgm}^{2}$ \\
$k$ & Column stiffness & $100 \mathrm{~N} \mathrm{mad}^{-1}$ \\
$N_{1}$ & Steering column-wheels gear ratio & 13.67 \\
$N_{2}$ & Motor-steering column gear ratio & 17 \\
$B_{v}$ & Steering wheel viscosity & $0.01 \mathrm{~N} \mathrm{mad}^{-1} \mathrm{~s}^{-1}$ \\
$B_{m}$ & Motor shaft viscosity & $0.0032 \mathrm{Nm} \mathrm{rad}^{-1} \mathrm{~s}^{-1}$ \\
$F_{c}$ & Coulomb friction force & $2.9 \mathrm{~N}^{-1}$ \\
$F_{n}$ & Normal force & $249.37 \mathrm{~N}^{-1}$ \\
$L$ & Patch length & $0.15 \mathrm{~m}^{-1}$ \\
$\sigma_{0}$ & Rubber longitudinal stiffness & $40 \mathrm{~m}^{-1}$ \\
$\sigma_{2}$ & Viscous relative damping & $0.0018 \mathrm{~s} \mathrm{~m}^{-1}$ \\
$P$ & Polynomial order & 13 \\
\hline
\end{tabular}

\section{TORQUES ACTING ON THE EPS SYSTEM}

The dynamical equations governing an EPS system are (see [17], [18] and [19]):

$$
\begin{aligned}
J_{v} \ddot{\theta}_{v} & =\tau_{v}-k\left(\theta_{v}-\theta_{s}\right)-B_{v} \dot{\theta}_{v} \\
J_{T} \ddot{\theta}_{s} & =-k\left(\theta_{s}-\theta_{v}\right)-N_{2}^{2} B_{m} \dot{\theta}_{s}-\sigma_{2} \frac{\dot{\theta}_{s}}{N_{1}^{2}}-\frac{\tau_{a}}{N_{1}}+U
\end{aligned}
$$

with $J_{T}=\left(J_{c}+N_{2}^{2} J_{m}+\frac{J_{w}}{N_{1}^{2}}\right), U=N_{2} u$ and $\theta_{v}, \theta_{s}$ and $\theta_{m}$ are, respectively, the steering wheel, the motor-shaft and the motor angles. The constants of the model are defined in Table I.

Let make the hypothesis that the stiffness $k$ of the steering column is infinity or that its effect can be compensated as proposed in [17]. In this case, it is possible to neglect the torsion of the column: i.e. $\theta_{v}-\theta_{s}=0$ and consider the equivalent steering wheel angle $\theta_{v}=\theta_{s}=\theta$. Summing Eq. (1) with Eq. (2), it is possible to obtain:

$$
J \ddot{\theta}+B \dot{\theta}+\frac{\tau_{a}}{N_{1}}=\tau_{v}+U
$$

where $J=J_{v}+J_{T}$ and $B=B_{v}+N_{2}^{2} B_{m}+\frac{\sigma_{2}}{N_{1}^{2}}$ are, respectively, the total inertia and the total viscosity of the system at the steering wheel level. From Eq. (3) arises that at stand hill, (or very low vehicle speeds, that is the common situation where the assistance acts), the most influencing torque, that opposes to the driver's torque, is the friction contact torque. For this reason, it is mandatory to have a realistic model of it and the following paragraph will be dedicated to the description of the adopted friction contact torque.

\section{A. Friction contact torque at stand-still}

The load torque $\tau_{a}$ is described by the Dahl's model ([20], [21], [22]), given by the following differential equation:

$$
\frac{\mathrm{d} F}{\mathrm{~d} \theta_{1}}=\sigma_{0}\left(1-\frac{F}{F_{c}} \operatorname{sgn}\left(\dot{\theta_{1}}\right)\right)
$$

where $\sigma_{0}$ is the rubber longitudinal stiffness coefficient, $F_{c}$ is the Coulomb friction force and $\theta_{1}=\theta / N_{1}$ is the steering wheel angle projected at the tire level. The friction force $|F|$ will never be larger than $F_{c}$, if its initial value is $|F(0)|<F_{c}$. To obtain a time-domain model, the following change of coordinates is required:

$$
\frac{\mathrm{d} F}{\mathrm{~d} t}=\frac{\mathrm{d} F}{\mathrm{~d} \theta_{1}} \frac{\mathrm{d} \theta_{1}}{\mathrm{~d} t}=\frac{\mathrm{d} F}{\mathrm{~d} \theta_{1}} \dot{\theta}_{1}=\sigma_{0}\left(1-\frac{F}{F_{c}} \operatorname{sgn}\left(\dot{\theta}_{1}\right)\right) \dot{\theta}_{1}
$$

The load torque $\tau_{a}$ in Eq. (3) is:

$$
\tau_{a}=F_{n} L F
$$

where $F_{n}$ and $L$ are, respectively, the normal force and the patch length.

For a constant speed $\dot{\theta}_{1}=\dot{\bar{\theta}}_{1}$, it is possible to obtain the following steady-state friction force $\bar{F}$ :

$$
\bar{F}=\sigma_{0} \bar{z}=F_{c} \operatorname{sgn}\left(\dot{\bar{\theta}}_{1}\right)
$$

This model is a first-order dynamic system, whose steadystate behaviour gives the Coulomb friction force, as shown in Eq. (7). For this model, it is possible to compute the solution of its differential equation in closed form for a sign-changing speed signal. Let consider a time interval $t \in\left[0, t_{1}\right]$, where the speed signal is positive and the system starts with the initial conditions, i.e. $F\left(\theta_{0}\right)=0$ and $\theta(0)=\theta_{0}$, the solution is:

$$
F(t)=F_{c}\left[1-e^{-\frac{\sigma_{0}}{F_{c}}\left(\frac{\theta(t)-\theta_{0}}{N_{1}}\right)}\right]
$$

If the speed signal becomes negative during a time interval $t \in\left[t_{1}, t_{2}\right]$, then the solution is:

$$
F(t)=F_{c}\left[-1+2 e^{\frac{\sigma_{0}}{F_{c}}\left(\frac{\theta(t)-\theta\left(t_{1}\right)}{N_{1}}\right)}-e^{\frac{\sigma_{0}}{F_{c}}\left(\frac{\theta(t)-2 \theta\left(t_{1}\right)}{N_{1}}\right)}\right]
$$

Note that this solution is evaluated starting from initial conditions, that guarantee the continuity of the solution at the time instant $t_{1}$, when the sign change of the speed happens. Finally, if the speed changes again in sign, during the time interval $t \in\left[t_{2}, T\right]$, the solution is:

$$
\begin{aligned}
& F(t)=F_{c}\left[1-2 e^{-\frac{\sigma_{0}}{F_{C}}\left(\frac{\theta(t)-\theta\left(t_{2}\right)}{N_{1}}\right)}+\right. \\
&\left.2 e^{-\frac{\sigma_{0}}{F_{C}}\left(\frac{\theta(t)+\theta\left(t_{1}\right)-2 \theta\left(t_{2}\right)}{N_{1}}\right)}-e^{-\frac{\sigma_{0}}{F_{C}}\left(\frac{\theta(t)+2 \theta\left(t_{1}\right)-2 \theta\left(t_{2}\right)}{N_{1}}\right)}\right]
\end{aligned}
$$

Details on the computation of these solutions are shown in [23]. For the purposes of this paper, the computation of the analytic solution is limited to three consecutive sign changes of the speed, but the proposed method can be applied even for more general cases. To compute the analytic solution, the time instant when the sign change takes place must be known. The friction force is only a function of the displacement and the sign of the relative velocity. This implies that it will depend only from the sign of the velocity, but not from its magnitude. 


\section{B. Perception of the load torque}

At stand still, the main force opposing to the driver's effort is the contact friction. In this situation, it is possible to assume that the assistance $U$ acts to compensate, first of all, a part of the contribution of the load torque $\tau_{a}$ in Eq. (3). Indeed at steady-state conditions:

$$
\frac{\tau_{a}}{N_{1}}=\tau_{v}+U
$$

In order to design the assistance amplification, the hypothesis that the driver's perception of the load torque obeys to the Stevens' power law ([24], [25], [26]) is done:

$$
\delta\left(\frac{\tau_{a}}{N_{1}}\right)^{n}=\tau_{v}
$$

where the term $\delta$ is the intensity magnitude of the friction torque and $n$ describes the rate of growth of the sensation of the stimulus and depends on the sensory modality of the driver (e.g. perception of force or perception of position). The value of $\delta$ is tuned to obtain a realistic value of the amplification torque, while to choose the value of $n$, it is mandatory to refer to the literature, concerning the control of the movement and to some experimental results.

During the steering movement, some authors ([27], [28]), show that drivers prefer exerting their control, basing on the transfer function between the friction contact load and the steering angle (position-oriented control), rather than the transfer function between the friction torque and the steering torque (torque-oriented control). This can be explained by the fact that the neuro-muscular system is better suited to control the steering angle than the exerted torque, thanks to the gamma $(\gamma)$ motor neurons, that adapt the length of the spindles according to the hand wheel angle [28].

In [24], authors determine experimentally the value of $n$ for both cases of torque-oriented and position-oriented control. For each case, they produce 3 experiments. In particular, for what concerns the position-oriented control, during the first experiment they require to 12 subjects to make a numerical estimation of the perceived magnitude of sensation. During the second one, they require to the same subjects to adjust the stimulus to produce a sensory magnitude equivalent to given numbers. Finally, they employ both magnitude estimation and magnitude production to develop a scale of perception of steady-state steering wheel angle. The value of the rate $n$ is determined by least square identification of the real data with Stevens' power law. They obtain an average value of $n=0.93$, that is $n<1$, meaning that the sensation of the angle grows at a slower rate than the angle. Note that results concerning the torque-oriented experiments indicate a rate of growth of the sensation $n>1$ to explain how human perceive the torque sensation in an opposite way.

Considering what has been highlighted to this point, in this paper a position-oriented control is considered and simulations are provided for values of $n \leq 1$.

\section{Relation with the assistance torque}

To obtain a relation between the assistance torque and the driver's torque in steady-state, Eq. (11) is substituted in
Eq. (12), as follows:

$$
\delta\left(\tau_{v}+U\right)^{n}=\tau_{v}
$$

By solving for $U$, it is possible to obtain:

$$
U=\sqrt[n]{\frac{\tau_{v}}{\delta}}-\tau_{v}
$$

When the dynamic terms of inertia and viscosity are included, the same closed-form solution for the assistance $U$ is not reachable, but it follows:

$$
J \ddot{\theta}+B \dot{\theta}+\delta\left(\frac{\tau_{a}}{N_{1}}\right)^{n}=\tau_{v}
$$

Let consider Eq. (3), we can express the assistance torque $U$, as the following difference:

$$
U=\frac{\tau_{a}}{N_{1}}-\delta\left(\frac{\tau_{a}}{N_{1}}\right)^{n}
$$

In this case, it is not possible to retrieve the corresponding value for the assistance torque in closed form, because it is necessary to solve Eq. (15) to obtain the corresponding values in Eq. (16). For this reason, it is necessary to introduce the optimization problem, shown in Section III.

To generalize the problem, the normalized form of the time is adopted:

$$
t^{*}=\frac{t}{T}
$$

where $T$ is the final simulation time, considered to execute the movement. Differentiating this time, it is possible to get:

$$
\mathrm{d} t^{*}=\frac{\mathrm{d} t}{T}
$$

In the new set of coordinates, the equations of the model becomes:

$$
\frac{J}{T^{2}} \frac{\mathrm{d}^{2} \theta}{\mathrm{d} t^{* 2}}+\frac{B}{T} \frac{\mathrm{d} \theta}{\mathrm{d} t^{*}}+\delta\left(\frac{\tau_{a}}{N_{1}}\right)^{n}=\tau_{v}^{*}
$$

The only time-invariant term in Eq. (19) is the friction torque, while the total inertia and the total viscosity decrease as slower the driver executes the movement.

\section{PROBLEM FORMULATION}

\section{A. Position-oriented control of the movement}

As discussed in Section I, many criteria used to explain the human movement are based on the minimization of a cost function. They can be divided in two main classes: dynamic and kinematic criteria.

Dynamic optimization criteria aim to minimize quantities like the energy or the power ([12], [13]), the torque and the torque change [14] exerted during a movement.

The most common kinematic criterion is based on the minimization of the jerk [29]. This function is widely used in literature to describe human pointing, because authors assume that the major goal of motor coordination is the production of the smoothest possible movement of the hand. Concerning the driving task, some authors formulated a position-oriented control ([27], [28]), that is in accordance with the kinematic optimization criteria. For this reason to 
obtain the profile of the booster curves for the dynamic case, an optimization problem basing on the minimization of the jerk function will be formulated in the following paragraph.

\section{B. Optimization problem}

The considered movement consists into bringing the steering wheel from $0 \mathrm{rad}$ to $\pi / 2 \mathrm{rad}$ and returning it to its initial position. The problem is to find the optimal trajectory $\hat{\theta}$, that minimizes the cost index $I$ :

$$
I=\int_{0}^{T}\left(\frac{\mathrm{d}^{3} \theta}{\mathrm{d} t^{3}}\right)^{2} \mathrm{~d} t
$$

under the constraint to have a driver's torque $\tau_{v}=0$ at the beginning and at the end of the movement.

In the normalized form of the time, the optimization problem becomes

$$
\min _{\hat{\theta}^{*}} \int_{0}^{1}\left(\frac{\mathrm{d}^{3} \theta^{*}}{\mathrm{~d} t^{* 3}}\right)^{2} \mathrm{~d} t^{*}
$$

with the boundary conditions on the trajectory

$$
\begin{array}{lll}
\theta^{*}(0)=0 & \theta^{*}(1)=0 & \theta^{*}(0.5)=\pi / 2 \\
\dot{\theta}^{*}(0)=0 & \dot{\theta}^{*}(1)=0 & \dot{\theta}^{*}(0.5)=0 \\
\ddot{\theta}^{*}(0)=0 & \ddot{\theta}^{*}(1)=0 &
\end{array}
$$

and on the driver's torque

$$
\tau_{v}(0)=0 \quad \tau_{v}(1)=0
$$

The condition $\tau_{v}(0)=0$ is guaranteed by the constraint to impose that the movement begins with speed and acceleration null and by the initial condition imposed on the friction torque $F(0)=0$. In the same way, to guarantee the condition $\tau_{v}(1)=0$, it is mandatory to impose that the load torque $\tau_{a}$ must be null at the final time $\tau_{a}(1)=0$, but this implies $F(1)=0$.

As the optimization procedure will use the analytic solution of the Dahl's friction torque, the constraint $F(1)=0$ can be imposed on the function in Eq. (10), obtained for $t \in\left[t_{2}, 1\right]$, as follows:

$$
\begin{aligned}
F(1) & =F_{C}\left[1-2 e^{-\frac{\sigma_{0}}{F_{C}}\left(\frac{\theta^{*}(1)-\theta^{*}\left(t_{2}^{*}\right)}{N_{1}}\right)}+2 e^{-\frac{\sigma_{0}}{F_{C}}\left(\frac{\theta^{*}(1)+\theta^{*}\left(t_{1}^{*}\right)-2 \theta^{*}\left(t_{2}^{*}\right)}{N_{1}}\right)}+\right. \\
& \left.-e^{-\frac{\sigma_{0}}{F_{C}}\left(\frac{\theta^{*}(1)+2 \theta^{*}\left(t_{1}^{*}\right)-2 \theta^{*}\left(t_{2}^{*}\right)}{N_{1}}\right)}\right]=0
\end{aligned}
$$

Using the boundary conditions imposed on the optimization problem, the values of $\theta^{*}(1)$ and $\theta^{*}\left(t_{1}^{*}\right)$, respectively, zero and $\pi / 2 \mathrm{rad}$, can be substituted in Eq. (24), and the only unknown variable becomes the angle $\theta^{*}\left(t_{2}^{*}\right)$ :

$$
\begin{aligned}
F(1) & =F_{c}\left[1-2 e^{\frac{\sigma_{0}}{F_{c}} \frac{\theta^{*}\left(t_{2}^{*}\right)}{N_{1}}}+2 e^{-\frac{\sigma_{0}}{F_{C}}\left(\frac{\frac{\pi}{2}-2 \theta^{*}\left(t_{2}^{*}\right)}{N_{1}}\right)}+\right. \\
& \left.-e^{-\frac{\sigma_{0}}{F_{C}}\left(\frac{\pi-2 \theta^{*}\left(t_{2}^{*}\right)}{N_{1}}\right)}\right]=0
\end{aligned}
$$

Setting $x=e^{\frac{\sigma_{0}}{F_{c}} \frac{\theta^{*}\left(t_{2}^{*}\right)}{N_{1}}}$, Eq. (25) can be written as the following second order algebraical equation:

$$
1-2 x+\left(2 e^{-\frac{\sigma_{0}}{F_{C}} \frac{\pi}{2} \frac{1}{N_{1}}}-e^{-\frac{\sigma_{0}}{F_{C}} \frac{\pi}{N_{1}}}\right) x^{2}=0
$$

The value for the angle $\theta^{*}\left(t_{2}^{*}\right)$ is obtained from the negative solution of this equation (the positive one is not compatible with the other constraints of the problem). Using this value, it possible to impose a further constraint on the trajectory, that guarantees the condition $F(1)=0$ :

$$
\theta^{*}\left(t_{2}^{*}\right)=\theta_{2}
$$

Nevertheless, it is not possible to predict a priori the instant $t_{2}^{*}$, when the steering wheel path may assume this value. To overcome this problem, an external dichotomy loop is used for the estimation of this value.

\section{Static optimization problem}

The problem to solve is a dynamic, non-linear, constrained optimization problem. To simplify the research of the optimal solution, it can be modified in a static, non-linear, constrained optimization problem.

Let make the hypothesis that among the set of the feasible solutions, the optimal one has the following polynomial form:

$$
\theta^{*}\left(t^{*}\right)=\sum_{k=0}^{P} a_{k} t^{* k}
$$

where $P$ is the order of the polynomial and $a_{k}$ is the single coefficient of the polynomial.

The derivatives of Eq. (28) provide the analytical expressions for $\dot{\theta}^{*}\left(t^{*}\right)$ and $\ddot{\theta}^{*}\left(t^{*}\right)$, that can be substituted in the Eq. (21) of the cost function, in the equations of the constraints (22), (23), (27) and in the movement equation (19), to retrieve the required torque in Eq. (16).

The optimization algorithm looks for the combinations of the polynomial $a_{k}$ with $k=0 \ldots P$, that minimize the cost function.

To impose the boundary conditions at the initial, intermediate and final time, it is sufficient to substitute the numerical values for the time in the polynomial equations. Remark that 9 boundary conditions are imposed; as consequence, the degree of the polynomial must be greater in order to allow to the optimization procedure to choose among different solutions.

\section{Simulation RESUlts}

The solution of this problem is obtained applying a convex programming algorithm, implemented with the software Matlab $2011^{\circledR}$

The produced trajectories, shown in Fig. 2, result in a twelfth order polynomial in time for the profile of the steering wheel angle and its derivatives. The cost function determines the form of the movement trajectory. The details are determined by the boundary conditions. Given this information and the duration of the movement, the trajectory of the steering wheel is specified in its entirety. No other information is required. As expected from an optimization that minimizes the jerk, the optimal trajectories are smooths and regulars. Note that the angular trajectory passes through the angle $\theta_{2}$ at the time $t_{2}=0.8 \mathrm{~s}$. This passage allows to obtain both the load and the driver's torques to be null at the final time.

Three examples of amplification torque are proposed. They 

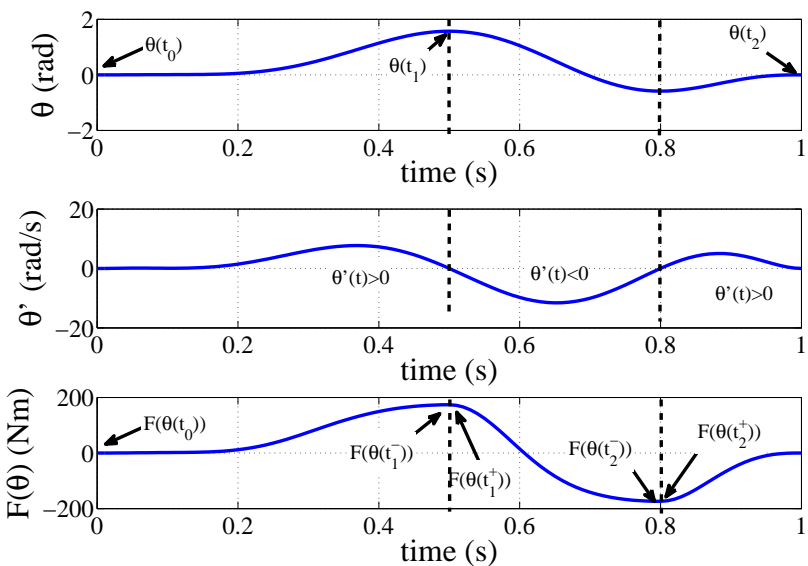

Fig. 2. Evolution of the angular and speed profile used to evaluate the analytic solution of the contact friction model.

are obtained by tuning the intensity magnitude $\delta$ and the rate of growth of the sensation of the stimulus $n$. For each case, two simulations are provided. They correspond, respectively, to steady-state case in Eq. (14) and to the general formulation in Eq. (16). The final simulation time is $T=15 \mathrm{~s}$, that provides a quasi-static case and a realistic booster curve. In Fig. 3, the coefficients are $n=1$ and $\delta=0.3$; in Fig. 4, $n=0.9$ and $\delta=0.3$, while in Fig. $5, n=0.5$ and $\delta=1$.

The predicted profiles of the booster curves are strongly dependent from the value of $n$. For the case in Fig. 3, the profile is linear in the stationary case; the hysteresis phenomena due to the influence of the inertia and the viscosity is evident when the transient state is considered.

For the cases $n<1$, shown in Figs. 4 and 5, at steady-state the curve has an exponential behaviour. This characteristic is more accentuated, as the coefficient $n$ diminishes. The amplification is almost negligible for driver's torques in the interval $-1 \leq \tau_{v} \leq+1 \mathrm{Nm}$, while it grows for more important values. The hysteresis effect is present when the optimization in transient state is carried out, due to the influence of the inertial and viscosity torques. Nevertheless, it is possible to conclude that the commonly used amplification curves correspond to the profile obtained with $n<1$. This means that the Stevens' coefficient for the rate of growth of the stimulus corresponds to the hypothesis of the positionoriented control of the movement.

\section{Conclusions}

The purpose of this paper is to provide some rationality the existing criteria used in EPAS systems. To this aim, the study of the main physical forces acting on the steering system is proposed. In particular, attention focused on a realistic model of the contact friction torque, because it represents the main torque that the assistance compensates.

The analysis was carried out in steady-state and transient conditions to provide a mathematical relation between the provided assistance and the driver's exerted torque. In steadystate, a relation in closed form between the assistance torque and the driver's torque was founded. For the transient case,

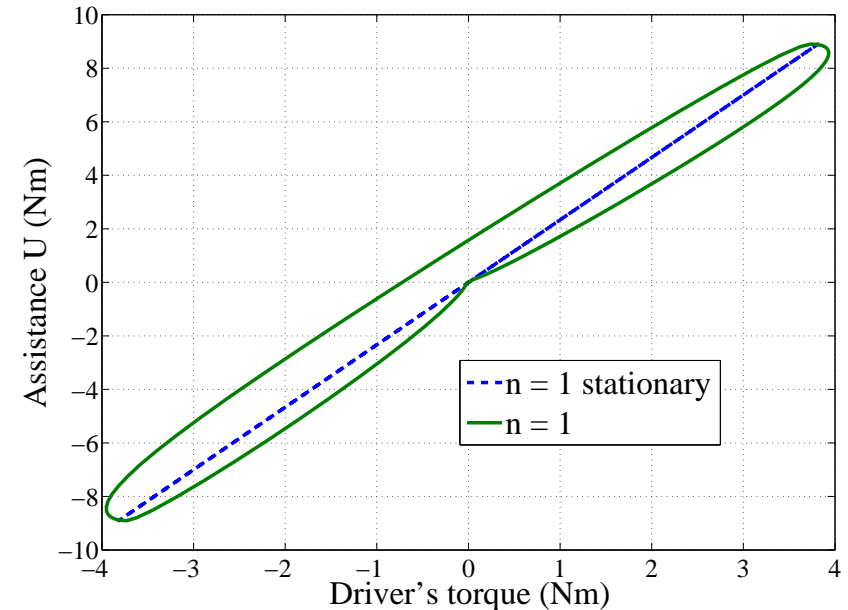

Fig. 3. Amplification torque obtained to compensate the friction torque with rate of the sensation stimulus $n=1$ in steady-state case (dotted) and dynamical one (full line)

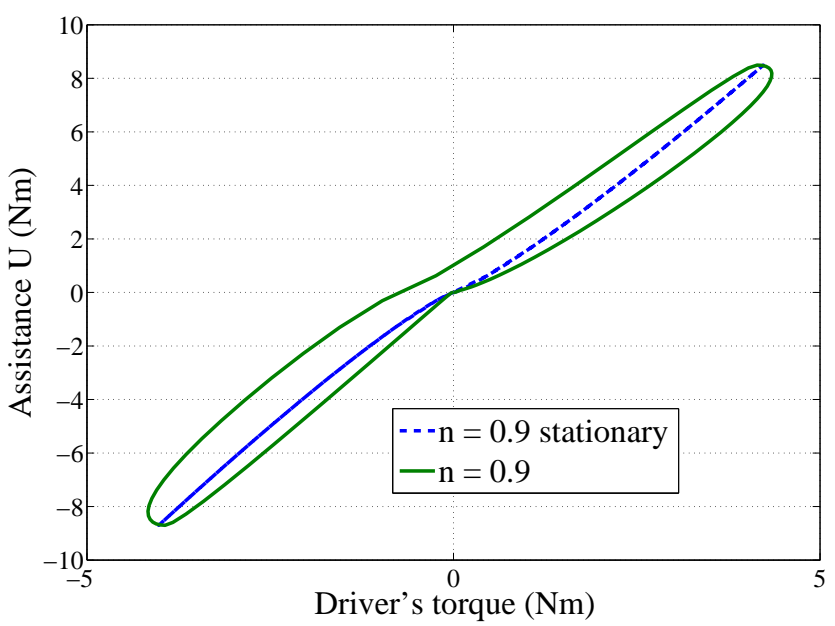

Fig. 4. Amplification torque obtained to compensate the friction torque with rate of the sensation stimulus $n=0.9$ in steady-state case (dotted) and dynamical one (full line)

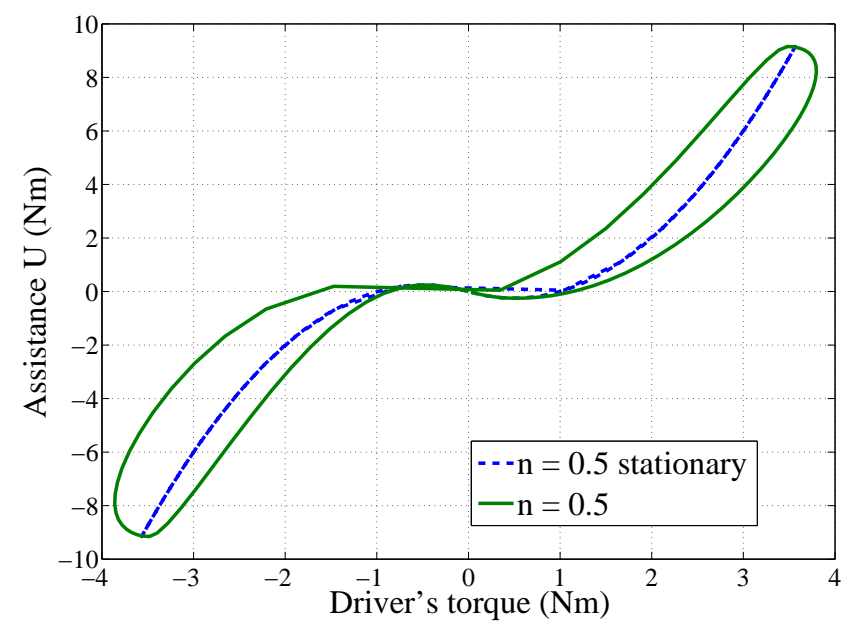

Fig. 5. Amplification torque obtained to compensate the friction torque with rate of the sensation stimulus $n=0.5$ in steady-state case (dotted) and dynamical one (full line) 
an optimization procedure is applied to reproduce the shape of the booster curves.

The study showed that the profile of the booster curve can be linked to minimization criteria based on the steering wheel jerk, coupled with the Stevens' power law. Moreover, the analysis showed that the profile of the predicted booster curves is strictly influenced by the rate of growth of the sensation of the stimulus, coming from the contact friction torque, but the commonly used booster curves are closed to those obtained, making the hypothesis of the positionoriented control of the movement.

\section{ACKNOWLEDGEMENTS}

This work was partially funded by the project VOLHAND ANR-09-VTT-14.

\section{REFERENCES}

[1] A. Zaremba, M. Liubakka, and R. Stuntz, "Control and steering feel issues in the design of an electric power steering system," in American Control Conference, 1998. Proceedings of the 1998, vol. 1, pp. 36-40, jun 1998.

[2] J. Wang, Q. Wang, and J. Liqiang, "Modeling and simulation studies on differential drive assisted steering for ev with four-wheelindependent-drive," in Vehicle Power and Propulsion Conference, 2008. VPPC '08. IEEE, pp. 1-7, sept. 2008.

[3] C. Canudas de Wit, "Fun-to-drive by feedback," in Decision and Control, 2005 and 2005 European Control Conference. CDC-ECC '05. 44th IEEE Conference on, p. 13, dec. 2005.

[4] C. Canudas de Wit, X. Claeys, and H. Bechart, "Stability analysis via passivity of the lateral actuator dynamics of a heavy vehicle," in Control Applications, 1999. Proceedings of the 1999 IEEE International Conference on, vol. 2, pp. 1371-1376, 1999.

[5] X. Claeys, C. Canudas de Wit, and H. Bechart, "Modeling and control of steering actuator for heavy duty vehicle," in European Control Conference, 1999.

[6] H. Zhang, Y. Zhang, J. Liu, J. Ren, and Y. Gao, "Modeling and characteristic curves of electric power steering system," in Power Electronics and Drive Systems, 2009. PEDS 2009. International Conference on, pp. 1390-1393, Nov. 2009.

[7] R. Chabaan, "Optimal control and gain scheduling of electrical power steering systems," in Vehicle Power and Propulsion Conference, 2009. VPPC '09. IEEE, pp. 53-59, Sept. 2009.

[8] H. B. Jiang, J. B. Zhao, H. M. Liu, and L. Chen, "Low-pass filter based automotive eps controller and comparative full-vehicle tests," in Control and Decision Conference, 2008. CCDC 2008. Chinese, pp. 4662-4665, July 2008.

[9] H. Tsung Hsien, Y. Chih Jung, H. Shih Rung, H. Tsung Hua, and L. Ming Chih, "Design of control logic and compensation strategy for electric power steering systems," in Vehicle Power and Propulsion Conference, 2008. VPPC' '08. IEEE, pp. 1-6, Sept. 2008.

[10] A. Burton, "Innovation drivers for electric power-assisted steering," Control Systems, IEEE, vol. 23, pp. 30-39, Dec. 2003.

[11] K. Ji Hoon and S. Jae Bok, "Control logic for an electric power steering system using assist motor," Mechatronics, vol. 12, no. 3, pp. 447-459, 2002.

[12] P. Li and R. Horowitz, "Control of smart exercise machines. i. problem formulation and nonadaptive control," Mechatronics, IEEE/ASME Transactions on, vol. 2, pp. 237-247, dec 1997.

[13] P. Li and R. Horowitz, "Control of smart exercise machines. ii. self-optimizing control," Mechatronics, IEEE/ASME Transactions on, vol. 2, pp. 248-258, dec 1997.

[14] K. Ohta, M. M. Svinin, Z. Luo, S. Hosoe, and R. Laboissire, "Optimal trajectory formation of constrained human arm reaching movements," Biological Cybernetics, vol. 91, pp. 23-36, 2004.

[15] S. E. Engelbrecht and J. P. Fernndez, "Invariant characteristics of horizontal-plane minimum-torque-change movements with one mechanical degree of freedom," Biological Cybernetics, vol. 76, pp. 321329, 1997.

[16] T. Flash and N. Hogans, "The coordination of arm movements: An experimentally confirmed mathematical model," Journal of neuroscience, vol. 5, pp. 1688-1703, 1985.
[17] J. Illán, V. Ciarla, and C. Canudas de Wit, "Oscillation annealing and driver/tire load torque estimation in electric power steering systems," in Control Applications (CCA), 2011 IEEE International Conference on, pp. 1100-1105, Sept. 2011.

[18] C. Canudas de Wit, S. Guegan, and A. Richard, "Control design for an electro power steering system: Part 1 the reference model," European Control Conference, Sept. 2001.

[19] C. Canudas de Wit, S. Guegan, and A. Richard, "Control design for an electro power steering system: Part 2 the control design," European Control Conference, Sept. 2001.

[20] P. Dahl and J. Ly, "Dynamic hysteresis modeling," AIAA Modeling and Simulation Technologies Conf., pp. 14-17, Aug. 2000.

[21] P. Dahl, "A solid friction model," Space and Missile Systems Organization Air Force Systems Command, May 1968.

[22] P. Dahl, "Solid friction damping of mechanical vibrations," AIAA Journal, vol. 14, pp. 1675-1682, 1976.

[23] V. Ciarla, V. Cahouet, C. Canudas de Wit, and F. Quaine, "Additional material for the paper: Genesis of booster curves in electric power assistance steering systems, submitted to itsc 2012," http://hal.archivesouvertes.fr/hal-00683760.

[24] A. C. Newberry, M. J. Griffin, and M. Dowson, "Driver perception of steering feel," Proceedings of the Institution of Mechanical Engineers Part D Journal of Automobile Engineering, vol. 221, no. 4, pp. 405415, 2007.

[25] M. Copelli, A. C. Roque, R. F. Oliveira, and O. Kinouchi, "Physics of psychophysics: Stevens and weber-fechner laws are transfer functions of excitable media," PHYSICAL REVIEW E, vol. 65, p. 060901, 2002.

[26] H. R. Bertollini G.P., "Applying driving simulation to quantify steering efforts preference as a function of vehicle speed," SAE Paper, 1999.

[27] W. Hoult and D. J. Cole, "A neuromuscular model featuring coactivation for use in driver simulation," Vehicle System Dynamics, vol. 46, pp. 175-189, 2008.

[28] A. J. Pick and D. J. Cole, "A mathematical model of driver steering control including neuromuscular dynamics," Journal of Dynamic Systems, Measurement, and Control, vol. 130, 2008.

[29] N. Hogan and D. Sternad, "On rhythmic and discrete movements: reflections, definitions and implications for motor control," Exp. Brain Res. 181, pp. 13-30, 2007. 